Corresponding author: Timothy.Tidwell@aruplab.com

(c) 2020 Tidwell et al. This article is distributed under the terms of the Creative Commons

Attribution-NonCommercial License, which permits reuse and redistribution, except for commercial purposes, provided that the original author and source are credited.

Ontology terms: bilateral cryptorchidism; bulbous nose; downturned corners of mouth; dysphagia; microcephaly; microretrognathia; mild intrauterine growth retardation; penoscrotal hypospadias; rhizomelia; severe global developmental delay; superior pectus carinatum

Published by Cold Spring Harbor Laboratory Press

doi:10.1101/mcs.a005728

\section{Novel de novo ARCN1 intronic variant causes rhizomelic short stature with microretrognathia and developmental delay}

\author{
Timothy Tidwell, ${ }^{1,4}$ Malia Deshotel, ${ }^{1,4}$ Janice Palumbos, ${ }^{2}$ Chris Miller, ${ }^{1}$ \\ Pinar Bayrak-Toydemir, ${ }^{1,3}$ and John C. Carey ${ }^{2}$
}

${ }^{1}$ ARUP Laboratories, Salt Lake City, Utah 84108, USA; ${ }^{2}$ Department of Pediatrics, University of Utah School of Medicine, Salt Lake City, Utah 84108, USA; ${ }^{3}$ Department of Pathology, University of Utah School of Medicine, Salt Lake City, Utah 84113, USA

\begin{abstract}
The archain 1 (ARCN1) gene encodes the coatomer subunit delta protein and is a component of the COPI coatomer complex, which is involved in retrograde vesical trafficking from the Golgi complex to the endoplasmic reticulum. Variants in ARCN1 have recently been associated with rhizomelic short stature with microcephaly, microretrognathia, and developmental delay. Here we report a 3.5-yr-old boy with microcephaly, global developmental delay, and multiple congenital abnormalities and the ARCN1-related syndrome caused by a novel de novo intronic variant. Whole-exome sequencing of the proband and his parents was utilized to determine the genetic origin of the patient's disorder and identified a de novo variant, NM_001655.5:c.654-15A>G, in the ARCN1 gene. Followup functional characterization of mRNA from the patient demonstrated that this variant creates a splicing defect of the ARCN1 mRNA. ARCN1-related syndrome represents an emerging disorder of developmental delay, and this report represents the sixth described patient. Despite the few instances reported in literature, the phenotype is consistent between our patient and previously reported individuals.
\end{abstract}

[Supplemental material is available for this article.]

\section{INTRODUCTION}

Global developmental delay (GDD), a common disorder affecting up to $3 \%$ of the population, occurs when children fail to meet normal developmental milestones in multiple domains (Mithyantha et al. 2017). There are many causes for developmental delay including maternal drug and alcohol exposure, birth complications, infections, and genetic conditions (Vissers et al. 2016). There is extreme genetic heterogeneity in GDD with more than 700 genes reported to be associated. Exome sequencing has been shown to be highly effective in the discovery of the genetic etiology of GDD and is even more effective in syndromic cases when additional findings are present (Srivastava et al. 2019). The yield for exome analysis is greatest when the parents of an affected individual are also sequenced allowing for the detection of de novo variants (de Ligt et al. 2012).

\footnotetext{
${ }^{4}$ These authors contributed equally to this work.
} 
The archain 1 (ARCN1) gene encodes the coatomer subunit delta protein and is a component of the COPI coatomer complex, which is involved in retrograde vesical trafficking from the Golgi complex to the endoplasmic reticulum (Tunnacliffe et al. 1996). This transport function is important for a wide variety of cell types and several multisystem disorders have been described in association with variants of genes in the COP1 complex (Watkin et al. 2015; Ge et al. 2016; DiStasio et al. 2017). Variants in ARCN1 have been reported in individuals with an autosomal dominantly inherited syndrome that includes rhizomelic short stature, with microcephaly, microretrognathia, and developmental delay (MIM: 617164) (Izumi et al. 2016; Reunert et al. 2019).

Here we present a report of an ARCN1-related syndrome caused by a novel de novo intronic variant. Functional analysis of the patient's mRNA demonstrated altered splicing consistent with loss of function of this allele.

\section{RESULTS}

\section{Clinical Presentation and Family History}

The patient was a 3.5-yr-old Caucasian/Peruvian/Native American boy with microcephaly, severe global developmental delay, and multiple congenital abnormalities. At birth he was documented to have a small ventricular septal defect (which was closed by $3 \mathrm{wk}$ ), a patent foramen ovale, rhizomelic shortening of extremities on clinical examination, pectus carinatum, and underdeveloped genitalia including severe penoscrotal hypospadias and cryptorchidism. His distinctive facial features recorded then included bulbous nasal tip, microretrognathia, and downturned corners of the mouth.

Gestational and birth history showed that the patient was delivered at 31 wk gestation by cesarean because of nonreassuring fetal testing, had Apgar scores of 6 and 8 , and was small for his gestational age (1244 g). His length at birth was $37 \mathrm{~cm}$, below the 10th centile, and his occipitofrontal circumference (OFC) was $27.5 \mathrm{~cm}$, which was just above the 10th. He developed spasticity and hyper-reflexia, likely related to a type III intraventricular hemorrhage $(\mathrm{IVH})$ seen on brain magnetic resonance imaging (MRI), which also showed white matter injury to both sides of the brain. The IVH was considered to be the underlying basis of the spastic quadriplegia. During early infancy he experienced severe aspiration and swallowing dysfunction causing G-tube dependence. The EEG was abnormal with excess background slowing, hemispheric asymmetry, and epileptiform features over the right hemisphere suggesting an underlying encephalopathy and seizure tendency. However, no seizures had occurred in the first $3 \mathrm{yr}$ of life. At 43 mo of age he had the onset of seizures; at 44 mo he was admitted to the hospital for status epilepticus. Since then the patient has gone on to have regular seizures on treatment and is considered to have intractable focal epilepsy. At $4 \mathrm{yr}$ and $8 \mathrm{mo}$ of age he was being treated with oxcarbazepine, Keppra, and Epidiolex and was starting the ketogenic diet. On genetics assessment at $3.5 \mathrm{yr}$, he had significant GDD. Skills included sitting with support, rolling both ways, downward scooting, and saying "mom" and "dad." He used a wheelchair for mobility and was not able to stand or walk. He was progressing slowly in his milestones and not regressing. Family history was noncontributory, and neither parent had short stature or his facial features. On physical examination, his height, weight, and OFC were all below the third centile; the distinctive facial features and rhizomelic shortening were again documented (Fig. 1A,B). He was enrolled in physical therapy, speech therapy, and occupational therapy since early infancy and has continued with these services. A previous genomic microarray identified a 95-kb loss at 12q23.2 including exons 1-4 of the NUP37 gene and exons 1-9 of the PARPBP gene, which were deemed nondiagnostic. Neither parent was tested for this deletion but analysis of exome coverage depth and single-nucleotide polymorphisms (SNPs) within this region suggests that the 
COLD SPRING HARBOR Molecular Case Studies
Novel de novo ARCN1 intronic variant
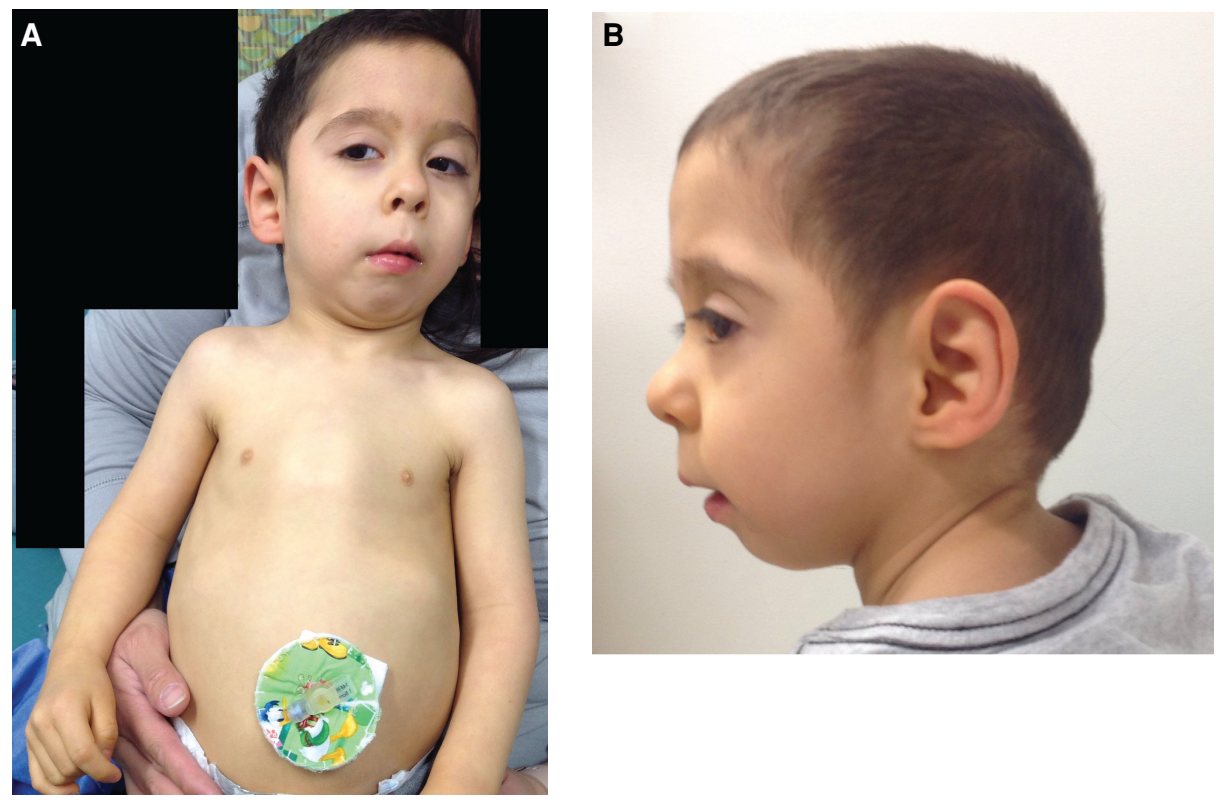

Figure 1. Patient data. (A) Patient at 3.5 yr old. (B) Profile of patient at 3 yr old. Patient has microcephaly, microretrognathia, pectus carinatum, and mild rhizomelic shortening of the limbs.

deletion was maternally inherited. A skeletal dysplasia panel had detected a heterozygous variant in HSPG2, c.9893 C > T, p. Pro3298Leu, which is associated with three skeletal disorders, none fitting his presentation; because he was heterozygous, the variant was maternally inherited and these conditions are autosomal recessive; this variant was not considered to be causative of his particular skeletal phenotype. Normal tests results included newborn metabolic screen, hearing screen, two skeletal surveys (Fig. 2), and chromosome analysis (46,XY).

\section{Genomic Analyses}

Although the history of prematurity and intraventricular hemorrhage (IVH) represented a potential explanation for his GDD, the other physical features, including the degree of short stature, the microretrognathia, microcephaly, rhizomelic shortening of extremities, pectus carinatum, and severe penoscrotal hypospadias, were not consistent with the complicated perinatal history as the cause, and therefore clinical exome sequencing was ordered.

Whole-exome sequencing of the proband and his parents identified a de novo variant, Chr 11(GRCh37):g.118455180A > G, (NM_001655.5:c.654-15A > G), in the ARCN1 gene (Table 1). Sanger sequencing confirmed this change to be present only in the proband and not the parents (Fig. 3). The c.654-15A > G variant creates a novel splice acceptor site by creating a new $A G$ nucleotide pair and computational splice site predictors indicate this new AG site may be utilized by splice machinery. When utilized, 14 novel nucleotides are inserted into the mRNA, which is predicted to shift the reading frame and create a premature stop codon p.Pro219Phefs*13.

\section{Functional Analyses}

To determine if the mRNA with the predicted alternative splicing isoform was detectable in the patient's sample, primers were designed to specifically target exon 4 of $A R C N 1$ with the partial 14-bp sequence (Fig. 4A) as predicted from the c.654-15A > G variant. RNA was extracted from the patient's whole blood and from three presumed wild-type controls, and 
A



B

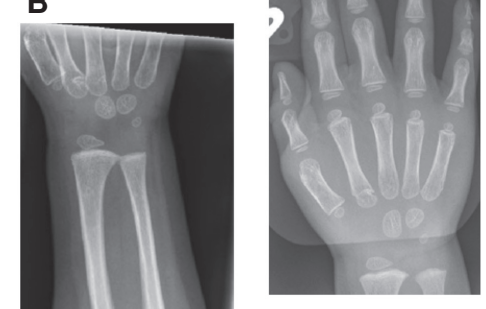

D

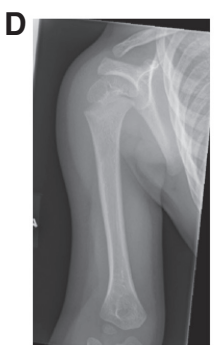

E

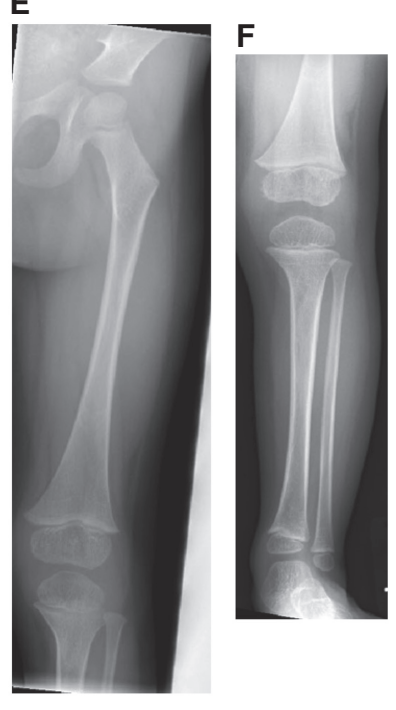

G

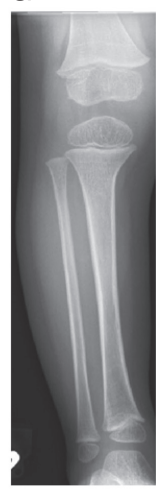

Figure 2. The composite figure depicts the skeletal survey performed at age $3 \mathrm{yr}$. The lateral spine $(A)$ and long bones $(B, D-G)$, including the humerus $(D)$, show normal contour. Of note, the clinically apparent rhizomelia is not seen on the radiographs of the upper limbs. The hands $(C)$ show hypoplasia of some distal phalanges and a pseudoepiphysis of the second metacarpal.

cDNA was generated. The cDNA samples were then tested with the sequencing primers, and only the patient's cDNA produced an amplicon (Fig. 4B). When the amplicons were sequenced and aligned to the NM_001655.5 reference sequence, a novel sequence was found between exons 4 and 5 (Fig. 4C, yellow highlighted region). Fourteen nucleotides of intron 4 are retained and were present on both forward and reverse sequencing (Fig. 4D).

\section{DISCUSSION}

Pathogenic loss-of-function variants in ARCN1 represent an emerging disorder of developmental delay and skeletal manifestations. The phenotype has proven to be relatively consistent among the five previously reported patients (Izumi et al. 2016; Reunert et al. 2019). The majority of patients demonstrate developmental delay, microcephaly, and short stature with rhizomelic shortening. Other less common manifestations include seizures, joint laxity, cleft palate, and need for tracheostomy. Transient defects in $\mathrm{N}$-glycosylation have also been reported in a patient with an ARCN1-related disorder; although this was not assessed in our patient, it provides a potential avenue to confirm the diagnosis. The history of prematurity and the subsequent IVH in our patient likely contributed to his GDD as he has early-onset spastic

\begin{tabular}{|c|c|c|c|c|c|c|c|c|c|}
\hline Gene & Chromosome & $\begin{array}{l}\text { HGVS DNA } \\
\text { reference }\end{array}$ & $\begin{array}{l}\text { HGVS } \\
\text { protein } \\
\text { reference }\end{array}$ & Variant type & $\begin{array}{l}\text { Predicted } \\
\text { effect }\end{array}$ & $\begin{array}{c}\text { dbSNP/ } \\
\text { dbVar } \\
\text { ID }\end{array}$ & Genotype & $\begin{array}{l}\text { Parent } \\
\text { of } \\
\text { origin }\end{array}$ & Observed effect \\
\hline ARCN1 & $\begin{array}{l}\text { NC_000011.9: } \\
\text { g.118455180A>G }\end{array}$ & $\begin{array}{l}\text { NM_001655.4: } \\
\text { c.654-15A>G }\end{array}$ & p.? & $\begin{array}{l}\text { Intronic } \\
\text { substitution }\end{array}$ & Frameshift & NA & Heterozygous & De novo & p.Pro219Phefs*13 \\
\hline
\end{tabular}




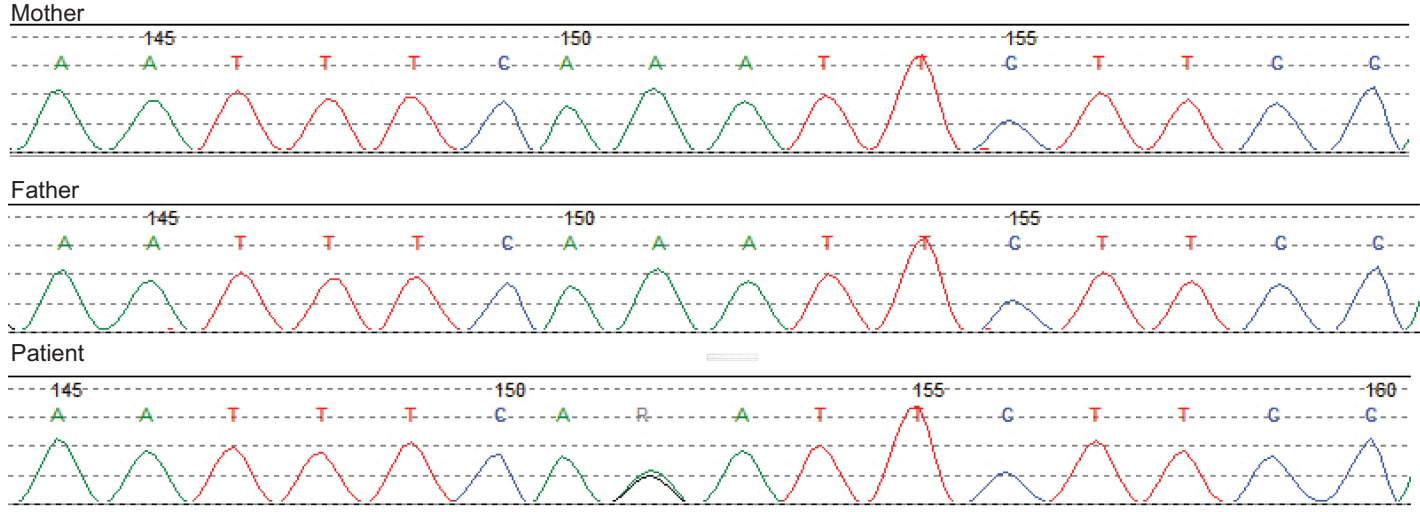

Figure 3. Sanger sequencing data from the patient and the patient's parents showing the de novo NM_001655.5(ARCN1):c.654-15A > G variant.

quadriplegia. However, his facial and skeletal manifestations cannot be explained by sequelae of IVH and fit the pattern described for ARCN1-related syndrome (Izumi et al. 2016).

Variants that affect splicing have historically been underrepresented but may account for up to $50 \%$ of all disease-causing variants (Wang and Cooper 2007). Identifying disease-

A

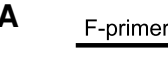

\begin{tabular}{|l|l|l|l|l}
\hline Exon 4 & & Exon 5 & Exon 6 & Exon 7 \\
\hline
\end{tabular}

predicted transcribed +14 bp sequence

B

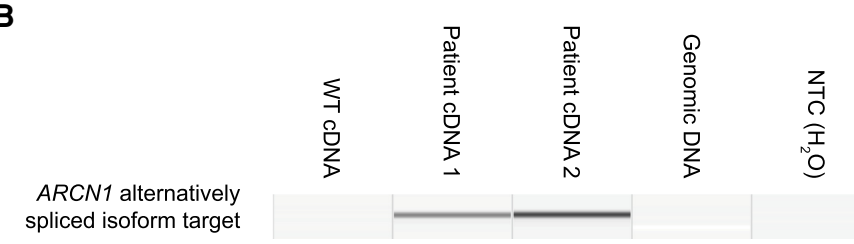

C

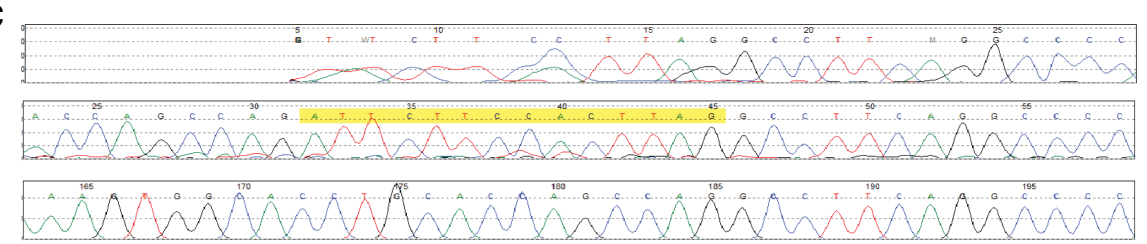

D Primer

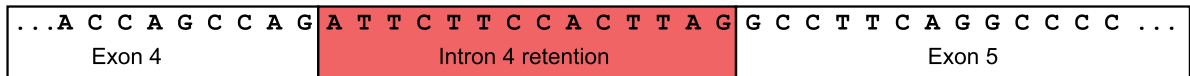

Figure 4. RNA studies. (A) To determine if the detected genomic variant altered RNA splicing, primers were designed to specifically amplify the predicted novel RNA isoform. (B) PCR products from the patient's sample and unrelated controls imaged with an eGene instrument. Only RNA extracted from the patient generated a PCR product indicating the presence of the predict RNA isoform. (C) Sanger sequence data. Patient sample forward reaction (top), reverse sequencing reaction (middle), and synthesized NM_001655.5 (ARCN1) RNA reference sequence (bottom). Nucleotides not present in the reference sequence are highlighted in yellow. (D) Schematic of new splice junction. The 14 retained nucleotides of intron 4 are highlighted in red. The partial primer site is represented by the blue bar. 
causing splice variants is complicated by lack of computational tools to identify new splice sites and the general unavailability of clinical methods to test for altered transcription (Spurdle et al. 2008). Out of the four algorithms, SpliceSiteFinder-like (Shapiro and Senapathy 1987; Zhang 1998), MaxEntScan (Yeo and Burge 2004), NNSPLICE (Reese et al. 1997), and GeneSplicer (Pertea et al. 2001), we routinely use for the assessment of intronic variants, only two (SpliceSiteFinder-like and MaxEntScan) predicted that the c.654-15A > G variant created a significant novel splice acceptor site. Splice site choice is a complicated process, and multiple splice acceptors can be utilized per exon (Scotti and Swanson 2016; Sibley et al. 2016; Cummings et al. 2017). Therefore, one limitation of the RNA testing methodology used is that we do not know what proportion of transcripts retain the intron. Based on the creation of a premature stop codon in this novel transcript it is predicted to undergo nonsense-mediated decay. This is expected to result in a deficiency of mature mRNA and protein, which would be consistent with previously identified ARCN1 variants but alternate mechanisms of disease cannot be ruled out. However, given the high clinical overlap with other reports of ARCN1-related disorders and because the detected variant occurred de novo in the patient, it is presumed that the splicing defect observed is sufficient to cause the disorder observed in the patient.

Exome sequencing is recommended as a first-tier test for patients with neurodevelopmental disorders including GDD (Srivastava et al. 2019). The clinical yield for exome testing is increased when trio sequencing is utilized for the identification of de novo variants (Retterer et al. 2016). Trio sequencing facilitated early identification of the de novo c.654$15 \mathrm{~A}>\mathrm{G}$ variant in our patient. Without the use of the parent's sequencing data, it would have been more difficult to identify the causative ARCN1 variant given the weak splice prediction and nucleotide conservation data.

To our knowledge, this patient represents the sixth documented case of an ARCN1-related disorder and the first disease-associated $A R C N 1$ splice variant. This case study further delineates the phenotypes associated with $A R C N 1$ and provides possible expansion of the phenotype. More case descriptions are required to understand the full scope of the phenotype and to provide prognostic and treatment-related recommendations.

\section{METHODS}

\section{Exome Sequencing}

Clinical exome sequencing was performed on the patient and his parents at ARUP Laboratories. Briefly, DNA was extracted from whole blood using the Chemagic MSM I instrument (PerkinElmer). XGen Exome Research Panel v1.0 (Integrated DNA Technologies) was used for exome capture and a NovaSeq 6000 (Illumina) was used for paired-end sequencing. A mean coverage depth of $199.6 \times$ was achieved for the proband and $95.5 \%$ of refseq-annotated exons were covered at a depth of $10 \times$ or greater. Full coverage data for all three individuals is available in Supplemental Table 1. Raw sequencing reads were aligned to the hg19 genome, and variants calls were filtered based on population frequency and inheritance. Reported variants were confirmed by targeted Sanger sequencing.

\section{RNA Analysis}

RNA was isolated from whole blood using the Maxwell RSC 48 simplyRNA Blood Kit (Promega). cDNA was generated with random hexamers using SuperScript III reverse transcriptase (Invitrogen), followed by RNase H treatment. Primers were designed with M13 sequences to specifically target the 14-bp alternatively spliced region directly downstream from exon 4 (indicated in bold, forward: 5'-TGTAAAACGACGGCCAGTACCAGCCAGAT 
$8_{\text {CSH }}^{\infty}$ C O L D S P R I N G H A R B O R Molecular Case Studies
Novel de novo ARCN1 intronic variant
Competing Interest Statement

The authors have declared no competing interest.

Received July 29, 2020; accepted in revised form October 16, 2020 .
TCTTCCACT-3'; reverse: 5'-CAGGAAACAGCTATGACCTTAGCACCCCTACGTCACTG-3'). PCR was performed with FailSafe premix J (Lucigen) and Accustart Taq (Quantabio) with a final primer concentration of $0.25 \mu \mathrm{M}$ in a $20 \mu \mathrm{L}$ reaction. Samples were amplified on SimpliAmp (ThermoFisher) thermalcyclers with a touchdown PCR cycle: an initial denaturation step of $95^{\circ} \mathrm{C}$ for $5 \mathrm{~min}$, followed by 10 cycles of $94^{\circ}$ for $30 \mathrm{sec}, 62^{\circ} \mathrm{C}$ (with an auto $\Delta-0.5^{\circ} \mathrm{C} / \mathrm{cycle}$ ) for $45 \mathrm{sec}$, and $72^{\circ} \mathrm{C}$ for $45 \mathrm{sec}$, followed by $35 \mathrm{cycles}$ of $94^{\circ} \mathrm{C}$ for $30 \mathrm{sec}$, $57^{\circ} \mathrm{C}$ for $45 \mathrm{sec}$, and $72^{\circ} \mathrm{C}$ for $45 \mathrm{sec}$, with a final extension step of $72^{\circ} \mathrm{C}$ for $7 \mathrm{~min}$ and an infinite hold of $4^{\circ} \mathrm{C}$. The resulting PCR products were visualized on the eGene (QIAGEN) and cleaned up with $4 \mu \mathrm{L}$ exoSAP-IT (ThermoFisher) on SimpliAmp thermalcyclers (ThermoFisher) with the following conditions: $37^{\circ} \mathrm{C}$ for $15 \mathrm{~min}, 85^{\circ} \mathrm{C}$ for $15 \mathrm{~min}$, and $4^{\circ} \mathrm{C}$ hold. The PCR amplicons were sequenced bidirectionally with M13 primers (forward: $5^{\prime}$-TGTAAAA CGACGGCCAGT-3'; reverse: 5'-CAGGAAACAGCTATGACC-3') and the BigDye Terminator v1.1 Cycle Sequencing kit (Applied Biosystems, ThermoFisher). Sequencing reaction products were purified with Sephadex G-50 Superfine beads (GE Life Sciences). Diluted purified sequencing products were detected on the ABI 3730xI DNA Analyzer (ThermoFisher) capillary electrophoresis instrument. The resulting sequencing files were analyzed with MutationSurveyor v5.1.2 (SoftGenetics) using the NM_001655.5 reference sequence.

\section{ADDITIONAL INFORMATION}

\section{Data Deposition and Access}

The ARCN1 variant has been submitted to ClinVar (https:/www.ncbi.nlm.nih.gov/clinvar/) under accession number SCV001142736.1. Consent was not obtained to submit exome sequencing data to a public repository.

\section{Ethics Statement}

We obtained signed consent from the parents of the proband under the University of Utah IRB_00007551, entitled Clinical Genetics Research program, renewed 05/15/2020. The family was enrolled and provided consent to participate in the collection of phenotypic and genetic data, October 8, 2019.

\section{Acknowledgments}

We thank the patient and his family for their participation.

\section{Author Contributions}

T.T., C.M., and P.B.-T. analyzed the exome data and provided diagnostic laboratory services. J.P. and J.C.C. provided clinical care, phenotyping, and clinical counseling. M.D. designed and performed the RNA analysis. T.T. and M.D. wrote the manuscript with the assistance of J.C.C. All authors provided comments and edits to the manuscript and agreed to the final contents.

\section{Funding}

There is no funding to disclose.

\section{REFERENCES}

Cummings BB, Marshall JL, Tukiainen T, Lek M, Donkervoort S, Foley AR, Bolduc V, Waddell LB, Sandaradura SA, O'Grady GL, et al. 2017. Improving genetic diagnosis in Mendelian disease with transcriptome sequencing. Sci Transl Med 9: eaal5209. doi:10.1126/scitranslmed.aal5209 
de Ligt J, Willemsen MH, van Bon BW, Kleefstra T, Yntema HG, Kroes T, Vulto-van Silfhout AT, Koolen DA, de Vries P, Gilissen C, et al. 2012. Diagnostic exome sequencing in persons with severe intellectual disability. N Engl J Med 367: 1921-1929. doi:10.1056/NEJMoa1206524

DiStasio A, Driver A, Sund K, Donlin M, Muraleedharan RM, Pooya S, Kline-Fath B, Kaufman KM, Prows CA, Schorry E, et al. 2017. Copb2 is essential for embryogenesis and hypomorphic mutations cause human microcephaly. Hum Mol Genet 26: 4836-4848. doi:10.1093/hmg/ddx362

Ge X, Gong H, Dumas K, Litwin J, Phillips JJ, Waisfisz Q, Weiss MM, Hendriks Y, Stuurman KE, Nelson SF, et al. 2016. Missense-depleted regions in population exomes implicate ras superfamily nucleotide-binding protein alteration in patients with brain malformation. NPJ Genom Med 1: 16036. doi:10.1038/npjgenmed .2016 .36

Izumi K, Brett M, Nishi E, Drunat S, Tan ES, Fujiki K, Lebon S, Cham B, Masuda K, Arakawa M, et al. 2016. ARCN1 mutations cause a recognizable craniofacial syndrome due to COPI-mediated transport defects. Am J Hum Genet 99: 451-459. doi:10.1016/j.ajhg.2016.06.011

Mithyantha R, Kneen R, McCann E, Gladstone M. 2017. Current evidence-based recommendations on investigating children with global developmental delay. Arch Dis Child 102: 1071-1076. doi:10.1136/archdis child-2016-311271

Pertea M, Lin X, Salzberg SL. 2001. GeneSplicer: a new computational method for splice site prediction. Nucleic Acids Res 29: 1185-1190. doi:10.1093/nar/29.5.1185

Reese MG, Eeckman FH, Kulp D, Haussler D. 1997. Improved splice site detection in Genie. J Comput Biol 4: 311-323. doi:10.1089/cmb.1997.4.311

Retterer K, Juusola J, Cho MT, Vitazka P, Millan F, Gibellini F, Vertino-Bell A, Smaoui N, Neidich J, Monaghan KG, et al. 2016. Clinical application of whole-exome sequencing across clinical indications. Genet Med 18: 696-704. doi:10.1038/gim.2015.148

Reunert J, Rust S, Grüneberg M, Seelhöfer A, Kurz D, Ocker V, Weber D, Fingerhut R, Marquardt T. 2019. Transient $\mathrm{N}$-glycosylation abnormalities likely due to a de novo loss-of-function mutation in the delta subunit of coat protein I. Am J Med Genet A 179: 1371-1375. doi:10.1002/ajmg.a.61190

Scotti MM, Swanson MS. 2016. RNA mis-splicing in disease. Nat Rev Genet 17: 19-32. doi:10.1038/nrg.2015.3

Shapiro MB, Senapathy P. 1987. RNA splice junctions of different classes of eukaryotes: sequence statistics and functional implications in gene expression. Nucleic Acids Res 15: 7155-7174. doi:10.1093/nar/15.17.7155

Sibley CR, Blazquez L, Ule J. 2016. Lessons from non-canonical splicing. Nat Rev Genet 17: 407-421. doi:10 1038/nrg.2016.46

Spurdle AB, Couch FJ, Hogervorst FB, Radice P, Sinilnikova OM, IARC Unclassified Genetic Variants Working Group. 2008. Prediction and assessment of splicing alterations: implications for clinical testing. Hum Mutat 29: 1304-1313. doi:10.1002/humu.20901

Srivastava S, Love-Nichols JA, Dies KA, Ledbetter DH, Martin CL, Chung WK, Firth HV, Frazier T, Hansen RL, Prock $L$, et al. 2019. Meta-analysis and multidisciplinary consensus statement: exome sequencing is a firsttier clinical diagnostic test for individuals with neurodevelopmental disorders. Genet Med 21: 2413-2421. doi:10.1038/s41436-019-0554-6

Tunnacliffe A, van de Vrugt H, Pensotti V, Radice P. 1996. The coatomer protein $\delta$-COP, encoded by the archain gene, is conserved across diverse eukaryotes. Mamm Genome 7: 784-786. doi:10.1007/ s003359900234

Vissers LE, Gilissen C, Veltman JA. 2016. Genetic studies in intellectual disability and related disorders. Nat Rev Genet 17: 9-18. doi:10.1038/nrg3999

Wang GS, Cooper TA. 2007. Splicing in disease: disruption of the splicing code and the decoding machinery. Nat Rev Genet 8: 749-761. doi:10.1038/nrg2164

Watkin LB, Jessen B, Wiszniewski W, Vece TJ, Jan M, Sha Y, Thamsen M, Santos-Cortez RL, Lee K, Gambin T, et al. 2015. COPA mutations impair ER-Golgi transport and cause hereditary autoimmune-mediated lung disease and arthritis. Nat Genet 47: 654-660. doi:10.1038/ng.3279

Yeo G, Burge CB. 2004. Maximum entropy modeling of short sequence motifs with applications to RNA splicing signals. J Comput Biol 11: 377-394. doi:10.1089/1066527041410418

Zhang MQ. 1998. Statistical features of human exons and their flanking regions. Hum Mol Genet 7: 919-932. doi:10.1093/hmg/7.5.919 


\section{COLD SPRING HARBOR Molecular Case Studies}

\section{Novel de novo $A R C N 1$ intronic variant causes rhizomelic short stature with microretrognathia and developmental delay}

Timothy Tidwell, Malia Deshotel, Janice Palumbos, et al.

Cold Spring Harb Mol Case Stud 2020, 6: a005728 originally published online November 5, 2020 Access the most recent version at doi: $10.1101 / \mathrm{mcs} .0005728$
Supplementary http://molecularcasestudies.cshlp.org/content/suppl/2020/11/10/mcs.a005728.D Material C1
References This article cites 21 articles, 2 of which can be accessed free at: http://molecularcasestudies.cshlp.org/content/6/6/a005728.full.html\#ref-list-1
License This article is distributed under the terms of the Creative Commons Attribution-NonCommercial License, which permits reuse and redistribution, except for commercial purposes, provided that the original author and source are credited.
Email Alerting Receive free email alerts when new articles cite this article - sign up in the box at the Service top right corner of the article or click here.

